\title{
Effects of the novel heat shock protein 90 inhibitor AUY922 in renal cell carcinoma $\mathrm{ACHN}$ and $786-\mathrm{O}$ cells
}

\author{
JINGJUAN ZHU ${ }^{1}$, YANBIAO ZHU ${ }^{2}$, WEIWEI QI ${ }^{1}$ and WENSHENG QIU ${ }^{1}$ \\ ${ }^{1}$ Department of Oncology, Affiliated Hospital of Qingdao University, Qingdao, \\ Shandong 266003; ${ }^{2}$ Department of General Surgery, Beicheng Hospital of Juye County, \\ Heze, Shandong 274900, P.R. China
}

Received May 17, 2014; Accepted February 13, 2015

DOI: $10.3892 / 01.2015 .3299$

\begin{abstract}
The aim of the present study was to investigate the effects of the heat shock protein (HSP)90 inhibitor AUY922 on the proliferation and migratory ability of renal cell carcinoma (RCC) cells. The expression of HSP90 was measured in vitro using western blotting and quantitative polymerase chain reaction in the ACHN and 786-O cell RCC lines, and also in the immortalized normal human proximal tubule epithelium HK-2 cell line. The effects of the time and concentration of AUY922 administration were investigated in the ACHN and 786-O cells, and the cell proliferation was measured using an MTT assay. A Transwell assay was performed to evaluate the migratory ability of ACHN cells following treatment with AUY922 at concentrations of 10,50 and $100 \mathrm{nM}$. Western blot analysis and reverse transcription polymerase chain reaction revealed that HSP90 mRNA and protein were overexpressed in the two RCC cell lines compared with the HK-2 cell line. AUY922 inhibited the proliferation of the ACHN and 786-O cells in a time- and concentration-dependent manner, and the migratory ability of the ACHN cells was markedly suppressed subsequent to treatment with AUY922. The present data suggest that the pathogenesis of human RCC may be mediated by HSP90. It was also indicated that the specific HSP90 inhibitor AUY922 plays a therapeutic role in the treatment of RCC, and therefore, HSP90 may be a selective target for molecular-targeted treatments of RCC.
\end{abstract}

\section{Introduction}

Renal cell carcinoma (RCC) accounts for $~ 90 \%$ of all kidney cancer cases, which in turn account for $2-3 \%$ of all cancers worldwide (1). RCC is the most lethal urological tumor and

Correspondence to: Dr Jingjuan Zhu, Department of Oncology, Affiliated Hospital of Qingdao University, 16 Jiangsu Road, Qingdao, Shandong 266003, P.R. China

E-mail: drzhujj@163.com

Key words: renal cell carcinoma, heat shock protein 90, AUY922, targeted therapy is the sixth leading cause of cancer-associated mortality in Western countries (1). Although the prevalence of RCC varies between populations and geographic regions, the incidence of RCC has increased steadily over previous years $(2,3)$. With the increased use of various imaging modalities, an increased number of RCC cases have been diagnosed at an early stage of disease. However, 20-30\% of patients with RCC demonstrate evidence of metastases at the time of the initial presentation (4). Although radical nephrectomy is an effective treatment for early-stage and local RCC, $30 \%$ of patients develop metastatic disease subsequent to surgery (5). Patients with metastatic RCC possess a poor prognosis, and therapeutic options are limited (6). The median survival of the cohort of patients with RCC enrolled in a previous study was only 1.5 years, with $<10 \%$ of patients surviving for five years (7). Therefore, the overall prognosis of patients with RCC is limited, indicating the requirement for improved therapies directed at potential molecular targets.

Heat shock protein 90 (HSP90) is a cellular molecular chaperone that is important for protein assembly, folding and stability (8). The client proteins of HSP90, including human epidermal growth factor receptor 2, Raf, mutant p53 and vascular endothelial growth factor (VEGF) receptor, play critical roles in oncogenesis (9). Numerous studies have demonstrated that HSP90 is overexpressed in solid tumors, and have reported that the inhibition of HSP90 exerts a promising therapeutic effect on certain cancers $(10,11)$. Previously, inhibition of HSP90 has been performed using the low molecular weight adenosine triphosphate (ATP)-competitive non-geldanamycin HSP90 inhibitor AUY922. This inhibitor has been speculated to offer advantages over ansamycin benzoquinone HSP90 inhibitors, including 17-allylamino-17-demethoxygeldanamycin (17-AAG), based on the independence of AUY992 from $\mathrm{NAD}(\mathrm{P}) \mathrm{H}$ :quinone oxidoreductase 1 metabolism, P-glycoprotein expression and favorable aqueous solubility (12). In the present study, the anti-tumor efficacy of the HSP90 inhibitor AUY922 was evaluated in the RCC ACHN and 786-O cell lines in vitro.

\section{Materials and methods}

Cell culture and reagents. The immortalized normal human proximal tubule epithelial HK-2 cell line was purchased from 
the American Type Culture Collection (Manassas, VA, USA). The human RCC 786-O and ACHN cell lines were obtained from the Cell Bank of Type Culture Collection of the Chinese Academy of Sciences (Beijing, China). The HK-2 cells were cultured in keratinocyte serum-free medium (Gibco Life Technologies, Carlsbad, CA, USA), and the 786-O and ACHN cells were cultured in RPMI-1640 medium (HyClone, Logan, UT, USA), supplemented with $10 \%$ fetal bovine serum (FBS) and $50 \mu \mathrm{g} / \mathrm{ml}$ streptomycin. All cells were cultured in a sterile incubator that was maintained at $37^{\circ} \mathrm{C}$ with an atmosphere of $5 \% \mathrm{CO}_{2}$. The HSP90 inhibitor AUY922 was purchased from Selleck Chemicals (Houston, TX, USA). The compounds were dissolved in dimethylsulfoxide (DMSO) to generate stock solutions at a concentration of $10 \mathrm{mM}$, and were then stored at $-20^{\circ} \mathrm{C}$. The mouse anti-human HSP90 monoclonal antibody was obtained from Abcam (Cambridge, MA, USA; catalog no., ab13492).

Western blot analysis. The cells were lysed in lysis buffer containing protease inhibitor. The protein concentration was determined using a bicinchoninic protein assay kit (Pierce Biotechnology, Inc., Rockford, IL, USA). In total, $30 \mu \mathrm{g}$ of the respective tissue proteins were separated by SDS-PAGE performed on $10 \%$ gels and then transferred onto polyvinylidene fluoride membranes (Millipore, Billerica, MA, USA). The membranes were blocked with 5\% non-fat milk and then incubated with mouse anti-human HSP90 monoclonal antibody (1:100; Abcam) and mouse anti-actin monoclonal antibody (catalog no. 0869100; 1:10,000; MP Biomedicals, Santa Ana, CA, USA). The membranes were washed three times for 10 min each using Tris-buffered saline comprising $50 \mathrm{mM}$ Tris (pH 7.4), $0.9 \% \mathrm{NaCl}$ and $0.05 \%$ Tween-20 (TBS-T), and were then incubated with phycoerythrin-conjugated secondary antibodies (donkey anti-mouse IgG H\&L; catalog no., ab7003; Abcam). The membranes were subsequently washed again with TBS-T three times, with each wash lasting for $10 \mathrm{~min}$. The target protein bands were visualized using the enhanced chemiluminescence method. All western immunoblot analyses were performed three times.

Total RNA extraction and complementary (c)DNA synthesis. Total RNA was isolated from the HK2, 786-O and ACHN cell lines using TRIzol Reagent (Invitrogen, Carlsbad, CA, USA), according to the manufacturer's instructions. The concentration and quality of the extracted total RNA was determined by measuring the optical density (OD) at 260 and $280 \mathrm{~nm}$, and then calculating the $\mathrm{OD}_{260}: \mathrm{OD}_{280}$ ratio. cDNA was synthesized from $2 \mu \mathrm{g}$ of total RNA using a Reverse Transcription System kit (Promega, Madison, WI, USA), according to the following protocol obtained from the reaction kit. Briefly, the samples were pre-incubated at $70^{\circ} \mathrm{C}$ for $10 \mathrm{~min}$, cooled on ice and then added to a reaction mixture consisting of $10 \mathrm{mmol} / 1$ deoxynucleotide triphosphate mixture, $25 \mathrm{mmol} / 1 \mathrm{MgCl}_{2}$, 15 units avian myoblastosis virus reverse transcriptase, $10 \mathrm{X}$ reverse transcription buffer, 0.5 units $\mathrm{RNasin}^{\circledR}$ and $0.5 \mu \mathrm{g}$ oligo-(dT) $)_{15}$ primer (all provided with the Reverse Transcription System kit). The reaction mixture was made up to a final volume of 201 and was incubated successively at $44^{\circ} \mathrm{C}$ for $15 \mathrm{~min}, 99^{\circ} \mathrm{C}$ for $5 \mathrm{~min}$ and $4^{\circ} \mathrm{C}$ for $5 \mathrm{~min}$. The cDNA was maintained at $20^{\circ} \mathrm{C}$ prior to use.
Reverse transcription-quantitative polymerase chain reaction (RT-qPCR). RT-qPCR was performed using SYBR Master Mix (Takara Bio Inc., Otsu, Japan) on an ABI Prism 7900HT Sequence Detection System (Applied Biosystems Life Technologies, Foster City, CA, USA). The human GAPDH gene was used as an endogenous control for sample normalization. The results were expressed as the fold change in expression relative to the expression of GAPDH. The PCR primers were as follows: GAPDH forward, 5'-GAGTCAACG GATTTGGTCGT-3' and reverse, 5'-GACAAGCTTCCCGTT CTCAG-3'; and HSP90 forward, 5'-TTGCTTCAGTGTCCC GGTGCG-3' and reverse, 5'-TGGTTGGTCTTGGGTCTG GGT-3'.

Cell viability measurement. Cell viability was analyzed using an MTT assay (Sigma-Aldrich, St. Louis, MO, USA). The cells were divided into three groups: the negative control group, which comprised cells treated with solvent DMSO; the blank control group, comprising cells treated with RPMI-1640 medium; and the experimental group. The cells of the experimental group were grown to $70-80 \%$ confluency in 96 well plates. AUY922 was then administered at a final concentration of $0.1,0.5,1,5,10,50$ or $100 \mathrm{nmol} / 1$ for treatment times of 24,48 or $72 \mathrm{~h}$. The blank group was treated with the addition of culture fluid. Subsequent to the reaction with the drugs for 24, 48 and $72 \mathrm{~h}$, the cells were then treated with $10 \mathrm{ml} /$ well MTT for $4 \mathrm{~h}$ at $37^{\circ} \mathrm{C}$. The cells underwent absorbance readings at $570 \mathrm{~nm}$ using a 96 -well microplate reader to obtain the $\mathrm{OD}$ values for the experimental $\left(\mathrm{OD}_{\text {experimental }}\right)$, blank control $\left(\mathrm{OD}_{\text {blank }}\right)$ and negative control $\left(\mathrm{OD}_{\text {negative }}\right)$ groups. The OD values were normalized to cells treated with $0 \mathrm{nmol} / \mathrm{l}$ of AUY922. The percentage of residual cell viability was calculated as follows:

$$
\text { Residual cell viability }(\%)=\frac{\left(\mathrm{OD}_{\text {experimental }}-\mathrm{OD}_{\text {blank }}\right)}{\left(\mathrm{OD}_{\text {negative }}-\mathrm{OD}_{\text {blank }}\right)} \times 100
$$

The assays were performed three times.

Cell migration assay. The motility capability of the cells was measured in vitro using Transwell chambers (Corning Inc., Corning, NY, USA). Four groups of ACHN cells $\left(10^{5}\right.$ per group, treated with 10,50 or $100 \mathrm{nmol} / \mathrm{l}$ AUY922, or blank control medium) were seeded onto the upper wells with serum-free medium. Medium supplemented with 20\% FBS was plated into the bottom wells to act as a chemoattractant. Subsequent to a 48-h period of incubation, the cells were fixed with methanol and stained with $1 \%$ crystal violet for $30 \mathrm{~min}$ at $37^{\circ} \mathrm{C}$. The cells that remained on the upper surface of the membranes were removed, while those on the lower surface were counted. Images of the migrated cells were captured under a microscope (CKX41SF; Olympus Corporation, Tokyo, Japan).

Statistical analysis. Data analyses were performed using SPSS software, version 15.0 (SPSS, Inc., Chicago, IL, USA). The results were expressed as the mean \pm standard deviation for continuous variables, and as the number and percentage for discrete variables. Differences in cell viability among groups was assessed using the $t$-test or two-way analysis of variance. 


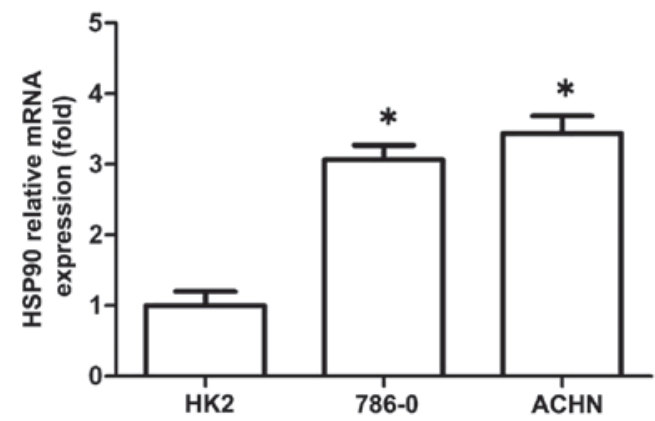

Figure 1. Reverse transcription polymerase chain reaction revealing the relative expression level of HSP90 mRNA in the renal cell carcinoma 786-O and ACHN cell lines and the immortalized normal human proximal tubule epithelial HK2 cell line. HSP90, heat shock protein 90.

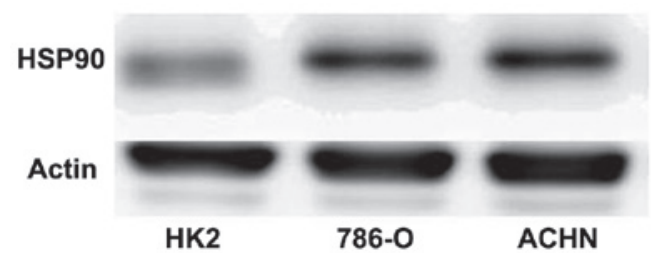

Figure 2. Western blot analysis revealing the expression of HSP90 in the renal cell carcinoma 786-O and $\mathrm{ACHN}$ cell lines and the immortalized normal human proximal tubule epithelial HK2 cell line. HSP90, heat shock protein 90

$\mathrm{P}<0.05$ was considered to indicate a statistically significant difference.

\section{Results}

Expression of the MRNA and protein of HSP9O in the RCC cell lines. RT-qPCR and western blot analysis were performed to detect the expression of the mRNA and protein of HSP90 in the RCC ACHN and 786-O and immortalized normal human proximal tubule epithelial HK-2 cell lines. As shown in Fig. 1, the 786-O and ACHN cell lines possessed higher HSP90 transcript levels compared with the HK-2 cell line. Similarly, the expression of the HSP90 protein was increased in the ACHN and 786-O cell lines compared with the HK-2 cell line (Fig. 2).

Effects of the HSP90 inhibitor AUY922 on cell growth. In order to determine whether HSP90 may be an effective therapeutic target for the treatment of RCC, the RCC cell lines were administered with AUY992 at a final concentrations of $0.1,0.5,1$, $5,10,50$ or $100 \mathrm{nmol} / 1$ for a treatment time of 24,48 or $72 \mathrm{~h}$, and the cell survival rate was measured using an MTT assay. As shown in Fig. 3, the MTT assay revealed that AUY922 significantly inhibited the proliferation of 786-O (Fig. 3A) and ACHN (Fig. 3B) cells in a time- and dose-dependent manner $(\mathrm{P}<0.05)$. The half-maximal growth inhibitory concentration $\left(\mathrm{IC}_{50}\right)$ of AUY992 in 786-O and ACHN cells after $72 \mathrm{~h}$ of treatment was $10.2 \mathrm{nM}$ and $7.6 \mathrm{nM}$, respectively.

Effect of the HSP90 inhibitor AUY922 on the migration of RCC cells. The present data revealed that the HSP90 inhibitor AUY922 inhibited the migration of the RCC ACHN cell line.
The Matrigel invasion assay revealed that the average number of cells migrating across the Matrigel-coated membrane in the negative control group was increased compared with the number in the AUY922-treated groups. The group of ACHN cells treated with 100 nM AUY992 yielded a decreased number of cells that migrated through the polycarbonate membrane compared with the group treated with 10 nM AUY992 $(\mathrm{P}<0.05)$ (Fig. 4). These results reveal that AUY992 performs a critical role in the inhibition of RCC cell migration.

\section{Discussion}

As the third most common genitourinary malignancy, RCC is not only a single oncological concept, but also a complex entity with respect to the etiology, histology and prognosis of the disease (13). Radical surgery remains the only potentially curative therapeutic option for RCC (14). Although an increased number of small renal masses are being detected, approximately one-third of patients present with metastatic disease (15). Metastatic RCC has demonstrated a poor response to chemotherapy and hormonal therapy. For patients with metastasized RCC that do not undergo complete resection, the five-year survival rate is $<10 \%$ (7). Significant advances in the understanding of the biology of RCC tumors have led to the development of molecular therapies targeting the VEGF and mammalian target of rapamycin (mTOR) pathways, resulting in a significant improvement in the overall survival rate and quality of life experienced by patients (16).

Although its molecular pathophysiology is not completely understood, an improved understanding of the molecular pathogenesis of RCC, particularly in genomics, proteomics and metabolomics, has attracted increasing attention during the last decade (17). These previous studies have allowed for the development of novel therapeutics that target the VEGF and mTOR pathways (18). HSP90 is the most abundant cytosolic HSP and regulates the maturation and stability of various proteins that are crucial for multiple cell signaling processes (19). In a variety of cancers, including hepatocellular carcinoma, breast cancer, non-small-cell lung cancer and prostate cancer, HSP90 is overexpressed and may contribute to tumor cell survival by mediating the maturation and stability of a variety of client proteins, such as VEGF and hypoxia inducible factor- $1 \alpha$ (HIF-1 $\alpha)$ (20). In the present study, RT-qPCR and western blot analysis were performed to detect the expression of the mRNA and protein of HSP90 in the RCC ACHN and 786-O cell lines, and also in the immortalized normal human proximal tubule epithelial HK-2 cell line. Western blot analysis and RT-qPCR revealed that the mRNA and protein of HSP90 were each overexpressed in the two RCC cell lines compared with the HK-2 cell line.

Small molecule inhibitors of the chaperone HSP90 are a growing class of clinically utilized anti-tumorigenic agents that have been collectively employed as an alternative means of targeting HIF-1 $\alpha$ (21). This focus on these inhibitors is due to their shared ability to disrupt the ATP-dependent chaperone activity of HSP90 and block the protein folding of HSP90 client proteins (21). Previous studies have revealed that the HSP90 inhibitors geldanamycin, 17-AAG and 17-dimethylaminoethylamino-17-demethoxygeldanamycin demonstrate 
A

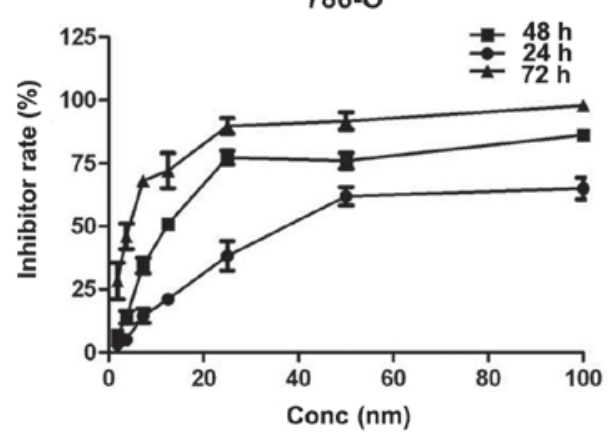

B

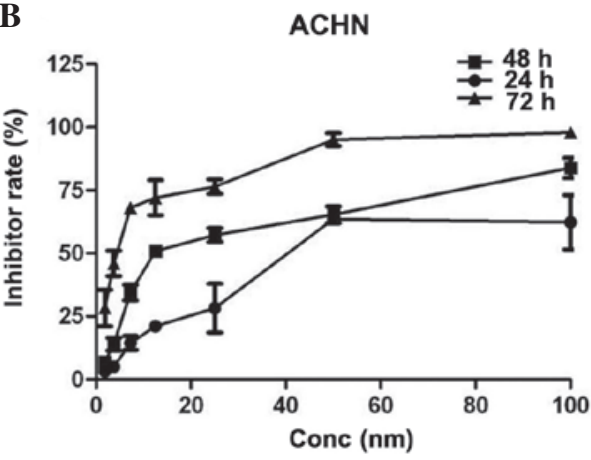

Figure 3. AUY922 inhibits the proliferation of the renal cell carcinoma 786-O and ACHN cell lines. The cell number was measured using an MTT assay. It was found that AUY922 inhibited cell proliferation in a concentration-dependent manner subsequent to 24,48 and $72 \mathrm{~h}$ of treatment in (A) $786-\mathrm{O}$ cells and (B) ACHN cells. Conc, concentration.
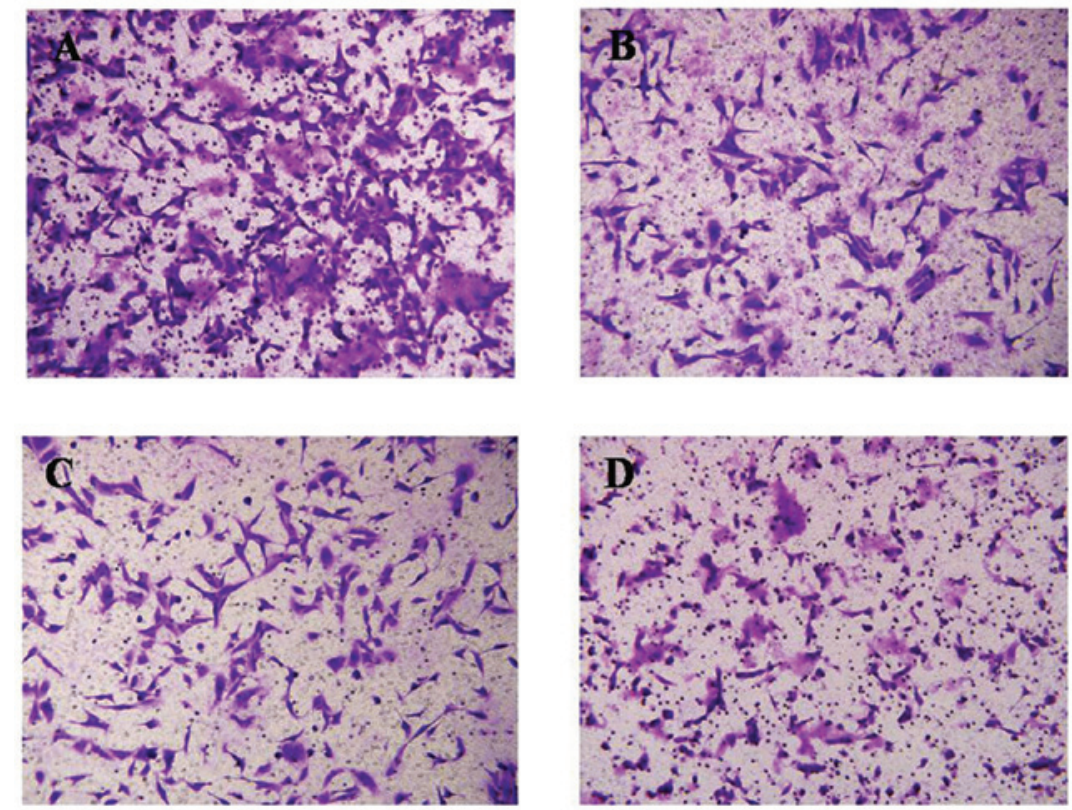

Figure 4. Inhibition of the migration of ACHN cells by AUY922. The images show the migration undergone by (A) negative control cells, (B) cells treated with $10 \mathrm{nM}$ AUY992, (C) cells treated with $50 \mathrm{nM}$ AUY992 and (D) cells treated with $100 \mathrm{nM}$ AUY992. Magnification, x20.

anti-tumorigenic and anti-angiogenic properties in vitro and in vivo in animal models, which is partly due to the ability of these agents to inhibit the function of $\operatorname{HIF}(22,23)$.

HSP90 inhibitors have been considerably improved. AUY922 is a member of the isoxazole HSP90-inhibitor family and inhibits ATPase activity with an $\mathrm{IC}_{50}$ of $30 \mathrm{nM}(24)$. This molecule is a non-geldanamycin analog that possesses the potential to cause prolonged target inhibition and at present, has not been associated with the same degree of hepatotoxicity as its geldanamycin counterparts. AUY922 exerts its effects by binding to the ATPase domain of the HSP90 N-terminal, preventing HSP90 from performing chaperone functions. This leads to the proteasomal degradation of numerous relevant client proteins. Administration of the single agent AUY922 has demonstrated potent preclinical anticancer activity in vitro and in vivo against a range of histological cell types (25-28). In the present study, RCC cells were treated with $1,2.5,5,10,25,50$ or $100 \mathrm{nM}$ of AUY922 for 24, 48 or $72 \mathrm{~h}$, respectively. The cell survival rate was measured using an MTT assay in order to assess the inhibitory effect of AUY922 on RCC cells. As shown in Fig. 3, the MTT assay revealed that AUY922 significantly inhibited the proliferation of 786-O (Fig. 3A) and ACHN (Fig. 3B) cells in a time- and dose-dependent manner $(\mathrm{P}<0.05)$. The $\mathrm{IC}_{50}$ of AUY992 in the 786-O and ACHN cells after $72 \mathrm{~h}$ was $10.2 \mathrm{nM}$ and $7.6 \mathrm{nM}$, respectively. AUY922 is able to inhibit the proliferation of various tumor cells according to previous studies, therefore the present results validate HSP90 as an important target in RCC treatment. The presumptive tumor suppressor function of the HSP90 inhibitor AUY922 in human RCC was further investigated in the current study using a Transwell assay. The results revealed that the cell migratory ability was significantly suppressed by AUY922. The migration assay revealed that the ACHN cells in the AUY922-treated groups were less likely to penetrate through the polycarbonate membrane compared with the negative control group. The data revealed this inhibition to be dose-dependent. Therefore, these results reveal a critical role of AUY922 in the inhibition of renal cancer cell migration. 
In conclusion, the present results validate HSP90 as an important target in RCC and treatment with the novel HSP90 inhibitor AUY922 may provide a promising strategy for antitumor therapy in RCC.

\section{References}

1. Ljungberg B, Campbell SC, Choi HY, Jacqmin D, Lee JE, Weikert S and Kiemeney LA: The epidemiology of renal cell carcinoma. Eur Urol 60: 615-621, 2011.

2. Miyamoto H, Miller JS, Fajardo DA, Lee TK, Netto GJ and Epstein JI: Non-invasive papillary urothelial neoplasms: The 2004 WHO/ISUP classification system. Pathol Int 60: 1-8, 2010.

3. Montironi R, Santinelli A, Pomante R, Mazzucchelli R, Colanzi P, Filho AL and Scarpelli M: Morphometric index of adult renal cell carcinoma. Comparison with the Fuhrman grading system. Virchows Arch 437: 82-89, 2000.

4. Janzen NK, Kim HL, Figlin RA and Belldegrun AS: Surveillance after radical or partial nephrectomy for localized renal cell carcinoma and management of recurrent disease. Urol Clin North Am 30: 843-852, 2003.

5. Jiang Z, Chu PG, Woda BA, Liu Q, Balaji KC, Rock KL and Wu CL: Combination of quantitative IMP3 and tumor stage: A new system to predict metastasis for patients with localized renal cell carcinomas. Clin Cancer Res 14: 5579-5584, 2008.

6. Zhao J, Huang X, Sun F, Ma R, Wang H, Shao K, Zhu Y, Zhou W, $\mathrm{Xu} \mathrm{Z}$ and Shen Z: Prognostic factors for overall survival with targeted therapy in Chinese patients with metastatic renal cell carcinoma. Can Urol Assoc J 8(11-12): E821-E827, 2014.

7. Patil S, Ishill N, Deluca J and Motzer RJ: Stage migration and increasing proportion of favorable-prognosis metastatic renal cell carcinoma patients: Implications for clinical trial design and interpretation. Cancer 116: 347-354, 2010.

8. Neckers L and Ivy SP: Heat shock protein 90. Curr Opin Oncol 15: 419-424, 2003

9. Maloney A and Workman P: HSP90 as a new therapeutic target for cancer therapy: the story unfolds. Expert Opin Biol Ther 2 : 3-24, 2002.

10. Trepel J, Mollapour M, Giaccone G and Neckers L: Targeting the dynamic HSP90 complex in cancer. Nat Rev Cancer 10 537-549, 2010

11. Powers MV and Workman P: Targeting of multiple signalling pathways by heat shock protein 90 molecular chaperone inhibitors. Endocr Relat Cancer 13 (Suppl 1): S125-S135, 2006.

12. Lee KH, Lee JH, Han SW, Im SA, Kim TY, Oh DY and Bang YJ: Antitumor activity of NVP-AUY922, a novel heat shock protein 90 inhibitor, in human gastric cancer cells is mediated through proteasomal degradation of client proteins. Cancer Sci 102: 1388-1395, 2011.

13. Jonasch E, Futreal PA, Davis IJ, Bailey ST, Kim WY Brugarolas J, et al: State of the science: an update on renal cell carcinoma. Mol Cancer Res 10: 859-880, 2012.
14. Janzen NK, Kim HL, Figlin RA and Belldegrun AS Surveillance after radical or partial nephrectomy for localized renal cell carcinoma and management of recurrent disease. Urol Clin North Am 30: 843-852, 2003.

15. Najjar YG and Rini BI: Novel agents in renal carcinoma: A reality check. Ther Adv Med Oncol 4: 183-194, 2012.

16. Mattei J, da Silva RD, Sehrt D, Molina WR and Kim FJ: Targeted therapy in metastatic renal carcinoma. Cancer Lett 343: 156-160, 2014

17. Santoni M, Pantano F, Amantini C, Nabissi M, Conti A, Burattini L, et al: Emerging strategies to overcome the resistance to current mTOR inhibitors in renal cell carcinoma. Biochim Biophys Acta 1845: 221-231, 2014.

18. Ngo TC, Wood CG and Karam JA: Biomarkers of renal cell carcinoma. Urol Oncol 32: 243-251, 2014

19. Xu Y, Zhang C, Chen D, Zhao J, Shen Z, Wu Y and Zhu Y: Effect of HSP90 inhibitor in pheochromocytoma PC12 cells: an experimental investigation. Tumour Biol 34: 4065-4071, 2013

20. Minet E, Mottet D, Michel G, Roland I, Raes M, Remacle J and Michiels C: Hypoxia-induced activation of HIF-1: Role of HIF-1alpha-Hsp90 interaction. FEBS Lett 460: 251-256, 1999.

21. Sato A, Asano T, Ito $K$ and Asano T: 17-Allylamino17-demethoxygeldanamycin and ritonavir inhibit renal cancer growth by inhibiting the expression of heat shock factor-1. Int J Oncol 41: 46-52, 2012.

22. Sanderson S, Valenti M, Gowan S, Patterson L, Ahmad Z, Workman P and Eccles SA: Benzoquinone ansamycin heat shock protein 90 inhibitors modulate multiple functions required for tumor angiogenesis. Mol Cancer Ther 5: 522-532, 2006.

23. Bisht KS, Bradbury CM, Mattson D, et al: Geldanamycin and 17-allylamino-17-demethoxygeldanamycin potentiate the in vitro and in vivo radiation response of cervical tumor cells via the heat shock protein 90-mediated intracellular signaling and cytotoxicity. Cancer Res 63: 8984-8995, 2003.

24. Pacey S, Gore M, Chao D, et al: A Phase II trial of 17-allylamino, 17-demethoxygeldanamycin (17-AAG, tanespimycin) in patients with metastatic melanoma. Invest New Drugs 30: 341-349, 2012.

25. Eccles SA, Massey A, Raynaud FI, et al: NVP-AUY922: A novel heat shock protein 90 inhibitor active against xenograft tumor growth, angiogenesis, and metastasis. Cancer Res 68: 2850-2860, 2008.

26. Moser C, Lang SA, Hackl C, Wagner C, Scheiffert E, Schlitt HJ, Geissler EK and Stoeltzing O: Targeting HSP90 by the novel inhibitor NVP-AUY922 reduces growth and angiogenesis of pancreatic cancer. Anticancer Res 32: 2551-2561, 2012.

27. Chatterjee M, Andrulis M, Stühmer T, et al: The PI3K/Akt signaling pathway regulates the expression of Hsp70, which critically contributes to Hsp90-chaperone function and tumor cell survival in multiple myeloma. Haematologica 98: 1132-1141, 2013.

28. Ueno T, Tsukuda K, Toyooka S, et al: Strong anti-tumor effect of NVP-AUY922, a novel Hsp90 inhibitor, on non-small cell lung cancer. Lung Cancer 76: 26-31, 2012. 